ITP-SB-96-22

INS-Rep.-1140

hep-th/9605102

May 1996

\title{
An explicit construction of Wakimoto realizations of current algebras
}

\author{
Jan de Boer ${ }^{\dagger}$ and László Fehér* \\ ${ }^{\dagger}$ Institute for Theoretical Physics \\ State University of New York at Stony Brook \\ Stony Brook, NY 11794-3840, USA \\ e-mail:deboer@insti.physics.sunysb.edu \\ * Institute for Nuclear Studies, University of Tokyo \\ Midori-cho, Tanashi-shi, Tokyo 188, Japan \\ e-mail:laszlo@ins.u-tokyo.ac.jp
}

\begin{abstract}
It is known from a work of Feigin and Frenkel that a Wakimoto type, generalized free field realization of the current algebra $\widehat{\mathcal{G}}_{k}$ can be associated with each parabolic subalgebra $\mathcal{P}=\left(\mathcal{G}_{0}+\mathcal{G}_{+}\right)$ of the Lie algebra $\mathcal{G}$, where in the standard case $\mathcal{G}_{0}$ is the Cartan and $\mathcal{P}$ is the Borel subalgebra. In this letter we obtain an explicit formula for the Wakimoto realization in the general case. Using Hamiltonian reduction of the WZNW model, we first derive a Poisson bracket realization of the $\mathcal{G}$-valued current in terms of symplectic bosons belonging to $\mathcal{G}_{+}$and a current belonging to $\mathcal{G}_{0}$. We then quantize the formula by determining the correct normal ordering. We also show that the affine-Sugawara stress-energy tensor takes the expected quadratic form in the constituents.
\end{abstract}




\section{Introduction}

In 1986 Wakimoto [1] found the following formula for the generating currents of $\widehat{s l}(2)_{k}$ :

$$
I_{-}=-p, \quad I_{0}=j_{0}-2(p q), \quad I_{+}=-\left(j_{0} q\right)+k \partial q+(p(q q)) .
$$

Here $j_{0}=-i \sqrt{2(k+2)} \partial \phi$, and the constituents are free fields whose commutation relations are encoded by the singular operator product expansions (OPEs)

$$
p(z) q(w)=\frac{-1}{z-w}, \quad \partial \phi(z) \partial \phi(w)=\frac{-1}{(z-w)^{2}} .
$$

The affine-Sugawara stress-energy tensor is quadratic in the free fields

$$
\frac{1}{2(k+2)}\left(\operatorname{tr} I^{2}\right)=-\frac{1}{2}(\partial \phi \partial \phi)-\frac{i}{\sqrt{2(k+2)}} \partial^{2} \phi-(p \partial q) \text {. }
$$

This result raised considerable interest because of its usefulness in computing correlation functions of conformal field theories [3, 4, 5, 6] and in describing the quantum Hamiltonian reduction of $\widehat{s l}(2)_{k}$ to the Virasoro algebra [7].

A detailed study of 'Wakimoto realizations' of arbitrary non-twisted affine Lie algebras was then undertaken in [3, , 1, 5, 8, 9, 10, 11] making use of the observation that at the level of zero modes (1.1) becomes the differential operator realization of $s l(2)$ given by

$$
I_{-} \rightarrow \frac{\partial}{\partial q}, \quad I_{0} \rightarrow-\mu_{0}+2 q \frac{\partial}{\partial q}, \quad I_{+} \rightarrow \mu_{0} q-q^{2} \frac{\partial}{\partial q}, \quad \forall \mu_{0} \in \mathbf{C} .
$$

It is well-known (e.g. [12]) that any simple Lie algebra, $\mathcal{G}$, admits analogous realizations in terms of first order differential operators of the form

$$
X \rightarrow \hat{X}=-F_{\alpha}^{X}(q) \frac{\partial}{\partial q_{\alpha}}+H_{\mu_{0}}^{X}(q), \quad \forall X \in \mathcal{G},
$$

where $F_{\alpha}^{X}, H_{\mu_{0}}^{X}$ are polynomials. In the principal case, $\alpha$ runs over the positive roots and $\mu_{0}$ is an arbitrary weight. The main idea [3, 5, 11] for generalizing (1.1) was to regard the differential operator realization of $\mathcal{G}$ as the zero mode part of the sought after realization of $\widehat{\mathcal{G}}_{k}$, which should be obtained by "affinization" where one replaces $q_{\alpha}$ and $p^{\alpha}$ by conjugate quantum fields and $\mu_{0}$ by a current that (in the principal case) belongs to the Cartan subalgebra of $\mathcal{G}$. Of course, one also needs to add derivative terms and find the correct normal ordering, which is rather nontrivial.

Feigin and Frenkel [3] demonstrated by indirect, homological techniques that the affinization can be performed for any simple Lie algebra $\mathcal{G}$. An explicit formula for the currents corresponding to the Chevalley generators of $s l(n)$ was given in [3] too, but the method does not lead to explicit formulas for all currents of an arbitrary $\mathcal{G}$ (or $\mathcal{G}=s l(n)$ ). Some explicit formulas were later obtained in [11] for any $\mathcal{G}$, but only for the Chevalley generators and

\footnotetext{
${ }^{1}$ We adopt the conventions of [2] for OPEs and for normal ordering.
} 
without complete proofs. The quadraticity of the affine-Sugawara stress-energy tensor in the free fields is not quite transparent in this approach.

In this letter we re-examine the construction of Wakimoto realizations. As our main result, we shall derive an explicit formula for the full $\mathcal{G}$-valued current in terms of finite group theoretic data. We also verify the quadraticity of the affine-Sugawara stress-energy tensor. We shall do this in the general case for which the Borel subalgebra of $\mathcal{G}$ that features in the principal case is replaced by an arbitrary parabolic subalgebra. The general case was previously investigated by Feigin and Frenkel (see the second article in [3]) in their homological approach, but we will use a different, direct method for the construction.

In our method we first derive a classical, Poisson bracket $(\mathrm{PB})$ version of the Wakimoto realization of $\widehat{\mathcal{G}}_{k}$. This will naturally result from a Hamiltonian reduction of the WZNW model based on the Lie group $G$ corresponding to $\mathcal{G}$. To motivate the construction in a simpler context, we shall also consider the finite dimensional version of the Hamiltonian reduction, which yields the $\mathrm{PB}$ analogue of the differential operator realization of $\mathcal{G}$. The $\mathrm{PB}$ realization of $\widehat{\mathcal{G}}_{k}$ is much closer to the desired OPE realization than the differential operator realization of $\mathcal{G}$ is, and it will not be very difficult to quantize it explicitly by normal ordering. The formula that we shall obtain (given in (4.6) together with (4.8)) should be useful in future applications of the Wakimoto realization.

In this letter we explain the construction outlined in the preceding paragraph and announce the main result; some proofs will be deferred to a subsequent paper [13].

In the rest of this section we collect some notions of Lie theory (see e.g. [14) that will be used. Let the step operators $E_{ \pm \alpha_{l}}$ and the Cartan elements $H_{\alpha_{l}}$, associated with the simple roots $\alpha_{l}$ for $l=1, \ldots, r=\operatorname{rank}(\mathcal{G})$, be the generators of the complex simple Lie algebra $\mathcal{G}$. For any $l=1, \ldots, r$, choose an integer $n_{l} \in\{0,1\}$ and determine the unique Cartan element $H$ for which $\left[H, E_{ \pm \alpha_{l}}\right]= \pm n_{l} E_{ \pm \alpha_{l}}$ (choices related by symmetries of the Dynkin diagram are equivalent). The eigenspaces of $H$ in the adjoint representation define a $\mathbf{Z}$-gradation of $\mathcal{G}$,

$$
\mathcal{G}=\oplus_{m} \mathcal{G}_{m} \quad\left[\mathcal{G}_{m}, \mathcal{G}_{n}\right] \subset \mathcal{G}_{m+n} \quad \text { with } \quad \mathcal{G}_{m} \equiv\{X \in \mathcal{G} \mid[H, X]=m X\} .
$$

Denoting the subspaces of positive/negative grades by $\mathcal{G}_{ \pm}$, we obtain the decomposition

$$
\mathcal{G}=\mathcal{G}_{-}+\mathcal{G}_{0}+\mathcal{G}_{+}
$$

We also consider a connected complex Lie group $G$ whose Lie algebra is $\mathcal{G}$ and its connected subgroups $G_{0, \pm}$ corresponding to the subalgebras $\mathcal{G}_{0, \pm}$, respectively. We then have the dense open submanifold $\check{G} \subset G$ of 'Gauss decomposable' elements,

$$
\check{G} \equiv\left\{g=g_{+} g_{0} g_{-} \mid g_{0, \pm} \in G_{0, \pm}\right\}
$$

which equals to $G_{+} \times G_{0} \times G_{-}$as a manifold since the decomposition of any $g \in \check{G}$ is unique. The parabolic subalgebra $\mathcal{P} \subset \mathcal{G}$ associated with the fixed set of integers $n_{l}$, and the corresponding parabolic subgroup $P \subset G$ are given by

$$
\mathcal{P} \equiv\left(\mathcal{G}_{0}+\mathcal{G}_{+}\right), \quad P=G_{0} G_{+},
$$


and have a semidirect product structure since $\left[\mathcal{G}_{0}, \mathcal{G}_{+}\right]=\mathcal{G}_{+}$. For $X, Y \in \mathcal{G}$, we shall denote an invariant scalar product $\langle X, Y\rangle$ simply as $\operatorname{Tr}(X Y)$ as if a matrix representation of $\mathcal{G}$ was chosen. Similarly, we denote say $\operatorname{Ad} g(X)$ as $g X g^{-1}$ for any $g \in G, X \in \mathcal{G}$. This notation is used purely for convenience, a choice of representation is never needed below. In the principal case, for which $n_{l}=1 \forall l, \mathcal{G}_{ \pm}$are the subalgebras generated by the positive/negative roots, $\mathcal{G}_{0}$ is the Cartan subalgebra, and the parabolic subalgebra is the Borel subalgebra. In the general case, the Lie algebra $\mathcal{G}_{0}$ can be decomposed into an abelian factor, say $\mathcal{G}_{0}^{0}$, and simple factors, say $\mathcal{G}_{0}^{i}$ for $i>0$, that are orthogonal with respect to $\operatorname{Tr}$,

$$
\mathcal{G}_{0}=\oplus_{i \geq 0} \mathcal{G}_{0}^{i}
$$

and $\mathcal{P}$ contains the Borel subalgebra. If $\psi$ and $\psi_{i}$ denote the highest roots of $\mathcal{G}$ and of $\mathcal{G}_{0}^{i}$ for $i>0$, then the dual Coxeter numbers of $\mathcal{G}$ and of $\mathcal{G}_{0}^{i}$ are respectively given by

$$
h^{*}=\frac{c_{2}(\mathcal{G})}{|\psi|^{2}} \quad \text { and } \quad h_{i}^{*}=\frac{c_{2}\left(\mathcal{G}_{0}^{i}\right)}{\left|\psi_{i}\right|^{2}} \quad \text { for } \quad i>0 .
$$

The quadratic Casimir $c_{2}(\mathcal{G})$ of $\mathcal{G}$ is defined by $\eta^{a b}\left[T_{a},\left[T_{b}, Y\right]\right]=c_{2}(\mathcal{G}) Y$ where $Y \in \mathcal{G}, \eta^{a b}$ is the inverse of $\eta_{a b}=\operatorname{Tr}\left(T_{a} T_{b}\right)$ for a basis $T_{a}$ of $\mathcal{G}$; and $c_{2}\left(\mathcal{G}_{0}^{i}\right)$ is defined analogously.

\section{Hamiltonian reduction in the finite group case}

We next derive a $\mathrm{PB}$ realization of the Lie algebra $\mathcal{G}$, which is the zero mode part of the classical Wakimoto realization of $\widehat{\mathcal{G}}_{k}$. The formula given by eq. (2.13) below will follow from a Hamiltonian symmetry reduction? of the holomorphic cotangent bundle $T^{*} G$ of $G$.

Using right translation by the elements $g \in G$, we have the identification

$$
T^{*} G=G \times \mathcal{G}=\{(g, J) \mid g \in G, J \in \mathcal{G}\} .
$$

The canonical symplectic form $\omega$ of $T^{*} G$ is then given by

$$
\omega=d \operatorname{Tr}\left(J d g g^{-1}\right)
$$

where $d$ denotes the exterior derivative. There are two commuting actions of $G$ on $T^{*} G$, which are induced by left and right translations on $G$ by any group element. The infinitesimal generators (the momentum map) that generate the left translations through $\mathrm{PBs}$ are just the components of $J$, while the infinitesimal generators of the right translations are the components of

$$
I=-g^{-1} J g .
$$

Both the components of $J$ and $I$ satisfy the Lie algebra $\mathcal{G}$ under the PB defined by $\omega$. We now consider a symmetry reduction using the action of the parabolic subgroup $P \subset G$ given by the left translations

$$
L_{p}:(g, J) \mapsto\left(p g, p J p^{-1}\right) \quad p \in P .
$$

\footnotetext{
${ }^{2}$ As a general reference, see e.g. [15].
} 
Decomposing $J$ as $J=\left(J_{-}+J_{0}+J_{+}\right)$according to (1.7), the momentum map corresponding to (2.4) is the projection $\left(J_{-}+J_{0}\right)$, and we define the reduction by imposing the constraints

$$
J_{-}=0 \quad \text { and } \quad J_{0}=\mu_{0}
$$

with some arbitrarily chosen $\mu_{0} \in \mathcal{G}_{0}$. The first class part of the constraints generates the subgroup $P\left(\mu_{0}\right) \subset P$, which is easily seen to have the structure

$$
P\left(\mu_{0}\right)=G_{0}\left(\mu_{0}\right) G_{+} \quad \text { with } \quad G_{0}\left(\mu_{0}\right) \equiv\left\{g_{0} \in G_{0} \mid g_{0} \mu_{0} g_{0}^{-1}=\mu_{0}\right\} .
$$

We are only interested in the dense open submanifold of the reduced phase space which results by restricting the group element $g \in G$ to belong to $\check{G} \subset G$ in (1.8). We find that a complete set of gauge invariant (i.e. $P\left(\mu_{0}\right)$ invariant) functions on the constrained manifold

$$
\left(T^{*} \check{G}\right)_{\text {const }}=\left\{\left(g_{+} g_{0} g_{-}, J_{+}+\mu_{0}\right) \mid g_{0, \pm} \in G_{0, \pm}, \quad J_{+} \in \mathcal{G}_{+}\right\}
$$

is given by the functions

$$
g_{-}, \quad j \equiv g_{0}^{-1}\left(g_{+}^{-1}\left(J_{+}+\mu_{0}\right) g_{+}-\mu_{0}\right) g_{0}, \quad j_{0} \equiv-g_{0}^{-1} \mu_{0} g_{0} .
$$

Furthermore, the mapping

$$
\left(g_{-}, j, j_{0}\right):\left(T^{*} \check{G}\right)_{\text {const }} \rightarrow G_{-} \times \mathcal{G}_{+} \times \mathcal{O}\left(-\mu_{0}\right)
$$

gives rise to a corresponding holomorphic diffeomorphism

$$
\left(g_{-}, j, j_{0}\right):\left(T^{*} \check{G}\right)_{\text {red }} \equiv P\left(\mu_{0}\right) \backslash\left(T^{*} \check{G}\right)_{\text {const }} \rightarrow T^{*} G_{-} \times \mathcal{O}\left(-\mu_{0}\right),
$$

where the identification $T^{*} G_{-}=G_{-} \times \mathcal{G}_{+}$is made by right translations and $\mathcal{O}\left(-\mu_{0}\right) \equiv$ $G_{0}\left(\mu_{0}\right) \backslash G_{0}$ is the co-adjoint orbit of $G_{0}$ through $-\mu_{0}$. The canonical symplectic form on $T^{*} G_{-} \times \mathcal{O}\left(-\mu_{0}\right)$ coincides with the induced symplectic form on the reduced phase space since

$$
\omega=d \operatorname{Tr}\left(J d g g^{-1}\right)=d \operatorname{Tr}\left(j d g_{-} g_{-}^{-1}\right)+d \operatorname{Tr}\left(\mu_{0} d g_{0} g_{0}^{-1}\right) \quad \text { on } \quad\left(T^{*} \check{G}\right)_{\text {const }} .
$$

Indeed, the two terms on the right hand side are recognized as the symplectic form of $T^{*} G_{-}$ and the symplectic form of $\mathcal{O}\left(-\mu_{0}\right)$.

The point of our construction is that, as it is invariant under the symmetry (2.4), $I$ survives the reduction. We have

$$
I=-\left(g_{+} g_{0} g_{-}\right)^{-1}\left(J_{+}+\mu_{0}\right)\left(g_{+} g_{0} g_{-}\right)=g_{-}^{-1}\left(-j+j_{0}\right) g_{-} \quad \text { on } \quad\left(T^{*} \check{G}\right)_{\text {const }} .
$$

Hence the result is the formula

$$
I\left(g_{-}, j, j_{0}\right)=g_{-}^{-1}\left(-j+j_{0}\right) g_{-}
$$

yielding a realization of the $\mathrm{PB}$ algebra of $\mathcal{G}$

$$
\{\operatorname{Tr}(X I), \operatorname{Tr}(Y I)\}=\operatorname{Tr}([X, Y] I) \quad X, Y \in \mathcal{G}
$$


in terms of the PB algebra of the constituents $g_{-}, j, j_{0}$. Introducing some global, holomorphic coordinates $q_{\alpha}$ on $G_{-}$and corresponding canonical coordinates $\left(q_{\alpha}, p^{\alpha}\right)$ on $T^{*} G_{-}$, $\operatorname{Tr}\left(j d g_{-} g_{-}^{-1}\right)=p^{\alpha} d q_{\alpha}$, the PB algebra of the constituents becomes

$$
\left\{q_{\alpha}, p^{\beta}\right\}=\delta_{\alpha}^{\beta}, \quad\left\{\operatorname{Tr}\left(X_{0} j_{0}\right), \operatorname{Tr}\left(Y_{0} j_{0}\right)\right\}=\operatorname{Tr}\left(\left[X_{0}, Y_{0}\right] j_{0}\right) \quad X_{0}, Y_{0} \in \mathcal{G}_{0}
$$

If we further let $V_{\alpha} \in \mathcal{G}_{-}$and $V^{\alpha} \in \mathcal{G}_{+}$denote dual bases, $\operatorname{Tr}\left(V_{\alpha} V^{\beta}\right)=\delta_{\alpha}^{\beta}$, then we have

$$
j(q, p)=N_{\alpha \beta}^{-1}(q) V^{\alpha} p^{\beta} \quad \text { with } \quad N^{\alpha \beta}(q) \equiv \operatorname{Tr}\left(V^{\beta} \frac{\partial g_{-}}{\partial q_{\alpha}} g_{-}^{-1}\right),
$$

since $\operatorname{Tr}\left(V_{\alpha} j\right)$ generates the action of $V_{\alpha} \in \mathcal{G}_{-}$on $T^{*} G_{-}$that comes from left translations, as is clear from (2.11). In summary, substituting $g_{-}(q)$ and $j(q, p)$ in (2.13), we obtain a realization of the PBs of $\mathcal{G}$ in terms of the simpler PBs in (2.15). Note that the differential operator realization of $\mathcal{G}$ on $P \backslash \breve{G}$ mentioned in (1.5) can be recovered by quantizing this $\mathrm{PB}$ realization. In the case when $\mu_{0}$ is a character of $\mathcal{G}_{0},(2.13)$ is in fact equivalent to the formula of the differential operator realization given by Theorems 2.1 and 2.2 in [12].

\section{Classical Wakimoto realizations of $\widehat{\mathcal{G}}_{k}$}

We here present the affine Lie algebraic analogue of the PB realization of the Lie algebra $\mathcal{G}$ derived in the preceding section. The PBs of $\widehat{\mathcal{G}}_{k}$ are given by

$$
\{\operatorname{Tr}(X I)(\sigma), \operatorname{Tr}(Y I)(\bar{\sigma})\}=\operatorname{Tr}([X, Y] I)(\sigma) \delta-K \operatorname{Tr}(X Y) \delta^{\prime} \quad X, Y \in \mathcal{G},
$$

where $I \in C^{\infty}\left(S^{1}, \mathcal{G}\right)$ is a $\mathcal{G}$-valued current and $\delta \equiv \delta(\sigma-\bar{\sigma})$ with $\sigma \in[0,2 \pi]$ being a coordinate on $S^{1}$. In fact, the proper analogue of formula (2.13) turns out to be

$$
I\left(q, p, j_{0}\right)=g_{-}^{-1}\left(-j+j_{0}\right) g_{-}+K g_{-}^{-1} g_{-}^{\prime}
$$

where $j$ is still given by (2.16) but the constituents are now promoted to be fields on $S^{1}$. More precisely, the $\mathcal{G}_{0}$-valued current $j_{0}$ and the fields $q_{\alpha}, p^{\beta}$ are subject to the PBs

$$
\begin{aligned}
\left\{\operatorname{Tr}\left(X_{0} j_{0}\right)(\sigma), \operatorname{Tr}\left(Y_{0} j_{0}\right)(\bar{\sigma})\right\} & =\operatorname{Tr}\left(\left[X_{0}, Y_{0}\right] j_{0}\right)(\sigma) \delta-K \operatorname{Tr}\left(X_{0} Y_{0}\right) \delta^{\prime} \quad X_{0}, Y_{0} \in \mathcal{G}_{0}, \\
\left\{q_{\alpha}(\sigma), p^{\beta}(\bar{\sigma})\right\} & =\delta_{\alpha}^{\beta} \delta(\sigma-\bar{\sigma}) .
\end{aligned}
$$

Using that $g_{-}$depends on the $q_{\alpha}$ by means of the chosen parametrization of $G_{-}$, one can write the PBs in (3.3) equivalently as

$$
\begin{aligned}
\left\{\operatorname{Tr}\left(V_{\alpha} j\right)(\sigma), \operatorname{Tr}\left(V_{\beta} j\right)(\bar{\sigma})\right\} & =\operatorname{Tr}\left(\left[V_{\alpha}, V_{\beta}\right] j\right)(\sigma) \delta, \\
\left\{\operatorname{Tr}\left(V_{\alpha} j\right)(\sigma), g_{-}(\bar{\sigma})\right\} & =-V_{\alpha} g_{-}(\sigma) \delta .
\end{aligned}
$$

\footnotetext{
${ }^{3}$ Summation on coinciding indices is understood throughout the text.
} 
The result that the PBs of $\widehat{\mathcal{G}}_{k}$ in (3.1) follow from the PBs of the constituents via the formula in (3.2) may be verified by a straightforward calculation. It can also be checked that the affine-Sugawara stress-energy tensor has the following quadratic structure:

$$
\frac{1}{2 K} \operatorname{Tr}\left(I^{2}\right)=\frac{1}{2 K} \operatorname{Tr}\left(j_{0}^{2}\right)-\operatorname{Tr}\left(j g_{-}^{\prime} g_{-}^{-1}\right)=\frac{1}{2 K} \operatorname{Tr}\left(j_{0}^{2}\right)-p^{\alpha} q_{\alpha}^{\prime} .
$$

It is natural to derive formula (3.2) by Hamiltonian symmetry reduction of the WZNW model [16] based on $G$, generalizing the construction presented in the finite group case. We take the phase space of the WZNW model to be the holomorphic cotangent bundle of the loop group $\widetilde{G}$ of $G$, which we realize as

$$
T^{*} \widetilde{G}_{K}=\left\{(g, J) \mid g \in C^{\infty}\left(S^{1}, G\right), \quad J \in C^{\infty}\left(S^{1}, \mathcal{G}\right)\right\} .
$$

This phase space has the symplectic form

$$
\omega_{K}=\int_{S^{1}} \delta \operatorname{Tr}\left(J \delta g g^{-1}\right)+K \int_{S^{1}} \operatorname{Tr}\left(\delta g g^{-1}\right)\left(\delta g g^{-1}\right)^{\prime}
$$

where $\delta$ denotes the functional exterior derivative (see e.g. [17]). Note that the 'left current' $J$ satisfies the analogue of (3.1) with opposite central term. The constraints we need to impose are formally identical to those in (2.5). Assuming that $g(\sigma) \in \check{G}$, on the constrained manifold we find the gauge invariant 'right current'

$$
I=-g^{-1} J g+K g^{-1} g^{\prime}=-\left(g_{+} g_{0} g_{-}\right)^{-1}\left(J_{+}+\mu_{0}\right)\left(g_{+} g_{0} g_{-}\right)+K\left(g_{+} g_{0} g_{-}\right)^{-1}\left(g_{+} g_{0} g_{-}\right)^{\prime},
$$

which is just $I$ in $(3.2)$ in terms of the gauge invariant objects

$$
j \equiv g_{0}^{-1}\left(g_{+}^{-1}\left(J_{+}+\mu_{0}\right) g_{+}-\mu_{0}-K g_{+}^{-1} g_{+}^{\prime}\right) g_{0}, \quad j_{0} \equiv-g_{0}^{-1} \mu_{0} g_{0}+K g_{0}^{-1} g_{0}^{\prime} .
$$

The PBs of $I$ and those of $g_{-}, j, j_{0}$ follow from this Hamiltonian reduction (see also [13]).

\section{Quantization of the classical Wakimoto realization}

Our goal now is to derive a quantum counterpart of the classical Wakimoto realization (3.2). As this classical realization was derived by means of a Hamiltonian reduction, there seem a priori to be two ways to quantize it. The first possibility would require us to write down a quantization of the phase space of the WZNW model and subsequently to implement a quantum Hamiltonian reduction. Although it might be very interesting to pursue this line of thought further, it seems to be rather cumbersome, and in addition in the case at hand it turns out to be relatively easy to directly quantize the classical Wakimoto realization. Therefore we will restrict ourselves to the latter method.

Since the classical Wakimoto realization expresses the currents of $\mathcal{G}$ in terms of currents in $\mathcal{G}_{0}$ and a set of coordinates and momenta that constitute the cotangent bundle $T^{*} \widetilde{G}_{-}$ of $\widetilde{G}_{-}=C^{\infty}\left(S^{1}, G_{-}\right)$, our philosophy will be to first quantize these objects by postulating OPEs for their generators, and subsequently to write down a normal ordered version of (3.2) 
in terms of these generators. The hard work lies in verifying that the currents defined in this way indeed satisfy the OPEs of the affine Lie algebra based on $\mathcal{G}$. This requirement will in addition fix the ambiguities that one has to deal with in normal ordering (3.2).

Fixing a basis $T_{a}$ of $\mathcal{G}$, the OPEs corresponding to (3.1) should read as

$$
\operatorname{Tr}\left(T_{a} I\right)(z) \operatorname{Tr}\left(T_{b} I\right)(w)=\frac{K \operatorname{Tr}\left(T_{a} T_{b}\right)}{(z-w)^{2}}+\frac{\operatorname{Tr}\left(\left[T_{a}, T_{b}\right] I\right)(w)}{z-w} .
$$

Replacing (3.1) with (4.1) amounts to replacing the PBs of the Fourier modes of the current with corresponding commutators, as is well-known. Naturally, the OPEs of the constituent coordinate and momentum fields are declared to be

$$
p^{\alpha}(z) q_{\beta}(w)=\frac{-\delta_{\beta}^{\alpha}}{z-w} .
$$

Decomposing the $\mathcal{G}_{0}$-valued current $j_{0}$ as $j_{0}=\sum_{i \geq 0} j_{0}^{i}$ according to (1.10), we postulate the OPEs of the current $j_{0}^{i}$ in $\mathcal{G}_{0}^{i}$ as

$$
\operatorname{Tr}\left(\pi_{0}^{i}\left(T_{a}\right) j_{0}^{i}\right)(z) \operatorname{Tr}\left(\pi_{0}^{i}\left(T_{b}\right) j_{0}^{i}\right)(w)=\frac{K_{0}^{i} \operatorname{Tr}\left(\pi_{0}^{i}\left(T_{a}\right) \pi_{0}^{i}\left(T_{b}\right)\right)}{(z-w)^{2}}+\frac{\operatorname{Tr}\left(\left[\pi_{0}^{i}\left(T_{a}\right), \pi_{0}^{i}\left(T_{b}\right)\right] j_{0}^{i}\right)(w)}{z-w}
$$

where $\pi_{0}^{i}: \mathcal{G} \rightarrow \mathcal{G}_{0}^{i}$ is the orthogonal projection onto $\mathcal{G}_{0}^{i}$. All other OPEs of the constituents are regular. Note that we have taken the central extension $K_{0}^{i}$ of $j_{0}^{i}$ to be a free parameter, to be determined from requiring (4.1), and that the properly normalized level parameters of $I$ and $j_{0}^{i}$ (which are integers in a unitary highest weight representation) are respectively given by

$$
k \equiv \frac{2 K}{|\psi|^{2}} \quad \text { and } \quad k_{0}^{i} \equiv \frac{2 K_{0}^{i}}{\left|\psi_{i}\right|^{2}} \quad \text { for } \quad i>0 .
$$

Notice now that the classical Wakimoto current in (3.2) is linear in the $p^{\alpha}$ and in $j_{0}$, but could contain arbitrary functions of the $q_{\alpha}$ if the coordinates were not chosen with care. However, we here only wish to deal with objects that are polynomial in the basic quantum fields, since those are easily defined in chiral conformal field theory (which is the same as the theory of vertex algebras [18]) by normal ordering. Below we will define a class of coordinates on $G_{-}$, the so-called 'upper triangular coordinates', in which the quantum Wakimoto current will be polynomial. The computations will also simplify considerably in these coordinates.

The only ordering problem in (3.2) arises from the term $-g_{-}^{-1} j g_{-}=-g_{-}^{-1} N_{\alpha \beta}^{-1}(q) p^{\beta} V^{\alpha} g_{-}$, for which we have to choose where to put the momenta $p^{\beta}$. We now choose to put them on the left, and replace any classical object $p f(q)$ by the normal ordered object $(p(f(q)))$. From the OPE

$$
p^{\alpha}(z) f(q(w))=\frac{-\partial^{\alpha} f}{z-w}
$$

we see that $\left(\left[p^{\alpha}, f(q)\right]\right)=-\partial \partial^{\alpha} f(q)=-\partial^{\beta} \partial^{\alpha} f(q) \partial q_{\beta}$. Hence, the difference between two normal orderings of the classical object $p f(q)$ will always be of the form $\Omega^{\beta}(q) \partial q_{\beta}$ for some

\footnotetext{
${ }^{4}$ The notations $\partial^{\alpha} f(q) \equiv \frac{\partial f(q)}{\partial q_{\alpha}}$ and $(\partial F)(z) \equiv \frac{\partial F(z)}{\partial z}$ are used for functions $f$ of $q$ and $F$ of $z$ from now on.
} 
function $\Omega^{\beta}$, and we should allow for an additional term of this type in quantizing (3.2). Altogether this leads to the following proposal for the quantum Wakimoto current:

$$
I=-\left(p^{\beta}\left(N_{\alpha \beta}^{-1} g_{-}^{-1} V^{\alpha} g_{-}\right)\right)+g_{-}^{-1} j_{0} g_{-}+K g_{-}^{-1} \partial g_{-}+g_{-}^{-1} \Omega^{\beta} g_{-} \partial q_{\beta} .
$$

Our main result will be to give the explicit form of the last term, which represents a quantum correction due to different normal orderings. The function $\Omega^{\beta}(q)$ is $\mathcal{G}$-valued and we inserted some factors of $g_{-}$around it for convenience.

To define the special coordinates in which our formula for $\Omega^{\beta}$ will be valid, we first introduce the matrix $R_{a}^{b}\left(g_{-}\right)$by

$$
g_{-} T_{a} g_{-}^{-1} \equiv R_{a}^{b}\left(g_{-}\right) T_{b} \quad g_{-} \in G_{-} .
$$

We call a system of global, holomorphic coordinates $q_{\alpha}$ on $G_{-}$polynomial if $R_{a}^{b}\left(g_{-}(q)\right)$ is given by a polynomial of the coordinates. The fact that $\operatorname{det} R=1$, which follows from the invariance of $\operatorname{Tr}$ and from the fact that $G_{-}$is topologically trivial, shows that $R_{a}^{b}\left(g_{-}^{-1}\right)$ is also polynomial in the $q_{\alpha}$. Furthermore, since one can evaluate $N^{\alpha \beta}(q)$ in $(2.16)$ using the adjoint representation, the definition implies that $N^{\alpha \beta}(q)$ is a polynomial, too. As it is non-vanishing and is given by a (complex) polynomial, the determinant of $N^{\alpha \beta}(q)$ must be a constant, from which we see that $N_{\alpha \beta}^{-1}(q)$ is a polynomial as well. Let now deg denote the gradation with respect to which the decomposition (11.7) was made, assume that the basis elements $V_{\alpha}$ of $\mathcal{G}_{-}$ have well-defined degree, and set $d_{\alpha} \equiv-\operatorname{deg}\left(V_{\alpha}\right)=\operatorname{deg}\left(V^{\alpha}\right)>0$. For polynomial coordinates $q_{\alpha}$ on $G_{-}$, let us assign degree $d_{\alpha}$ to $q_{\alpha}$. By definition, upper triangular coordinates are such polynomial coordinates for which $N^{\alpha \beta}(q)$ is given by a homogeneous polynomial of degree $\left(d_{\beta}-d_{\alpha}\right)$ with respect to this assignment of the degree.

In upper triangular coordinates $N^{\alpha \beta}(q)$ obviously vanishes unless $d_{\beta} \geq d_{\alpha}$, which explains the name and implies that $N_{\alpha \beta}^{-1}(q)$ is also a polynomial of degree $\left(d_{\beta}-d_{\alpha}\right)$. The most obvious examples of upper triangular coordinates are the 'graded exponential coordinates', given by $g_{-}(q)=\exp \left(\sum_{\alpha} q_{\alpha} V_{\alpha}\right)$. One can also take products of graded exponential coordinates, by distributing the set $\left\{V_{\alpha}\right\}$ over disjoint subsets $S_{\mathcal{I}}$ and taking $g_{-}(q)=\prod_{\mathcal{I}} \exp \left(\sum_{\alpha \in S_{\mathcal{I}}} q_{\alpha} V_{\alpha}\right)$. If $G=S L(n)$, there are even simpler coordinates where the $q_{\alpha}$ are matrix elements, namely $g_{-}(q)=\mathbf{1}_{n}+\sum_{\alpha} q_{\alpha} V_{\alpha}$ with $\left(V_{\alpha}\right)_{l k}=\delta_{i l} \delta_{j k}$ for some $i>j$. To check the upper triangularity property in these examples, it is useful to think of $g_{-}(q)$ as a polynomial in the $q_{\alpha}$ and the $V_{\alpha}$ which are declared to have degrees $d_{\alpha}$ and $-d_{\alpha}$, respectively. Then $g_{-}(q)$ has 'total degree' zero, and $N^{\alpha \beta}(q) V_{\beta}$ has total degree $-d_{\alpha}$, implying that $N^{\alpha \beta}(q)$ has degree $\left(d_{\beta}-d_{\alpha}\right)$.

Now we are ready to state our main result:

Given a system of upper triangular coordinates $q_{\alpha}$ on $G_{-}$, the current I defined in (4.0) satisfies the OPE given in (4.1) if (i) $2 K_{0}^{0}=|\psi|^{2}\left(k+h^{*}\right)=\left|\psi_{i}\right|^{2}\left(k_{0}^{i}+h_{i}^{*}\right)$ for $i>0$, and (ii) $\Omega^{\beta}$ is given by the following $\mathcal{G}_{-}$-valued object

$$
\Omega^{\beta}=N^{\lambda \rho} \partial^{\beta} N_{\gamma \lambda}^{-1}\left[V^{\gamma}, V_{\rho}\right] .
$$

Furthermore, $\Omega^{\beta}$ is uniquely determined by (4.1) up to trivial redefinitions of the momenta $p^{\beta}$ in (4.0),

$$
p^{\beta} \rightarrow p^{\beta}+\left(\partial^{\beta} A^{\gamma}-\partial^{\gamma} A^{\beta}\right) \partial q_{\gamma}
$$


with an arbitrary polynomial $A^{\gamma}(q)$. Finally, the stress-energy tensor for the current I takes the form of a free stress-energy tensor for $p^{\beta}, q_{\beta}$ and a sum of improved stress energy tensors for the currents $j_{0}^{i}$ with values in $\mathcal{G}_{0}^{i}$,

$$
\frac{1}{2 y} \operatorname{Tr}\left(I^{2}\right)=-p^{\beta} \partial q_{\beta}+\frac{1}{2 y} \sum_{i \geq 0} \operatorname{Tr}\left(j_{0}^{i} j_{0}^{i}\right)+\frac{1}{y} \operatorname{Tr}\left(\left(\rho_{\mathcal{G}}-\rho_{\mathcal{G}_{0}}\right) \partial j_{0}\right)
$$

where $y \equiv \frac{1}{2}|\psi|^{2}\left(k+h^{*}\right)$ and $\rho_{\mathcal{G}}-\rho_{\mathcal{G}_{0}}=\frac{1}{2}\left[V^{\alpha}, V_{\alpha}\right]$ is half the sum of those positive roots of $\mathcal{G}$ that are not roots of $\mathcal{G}_{0}$.

The proof of the above statements consists of an explicit calculation of the OPEs of $I$ with itself and of the normal ordered product $\operatorname{Tr}\left(I^{2}\right)$. This calculation is relatively straightforward but rather long, and therefore we do not present it here (see [13]). The relationship between $k, k_{0}^{i}$ and $K_{0}^{0}$ given in condition (i) above was also found by Feigin and Frenkel, although the correct normalization factors are missing in Proposition 4 in the second article of [3] concerning those cases for which $\left|\psi_{i}\right|^{2} \neq|\psi|^{2}$. We checked that (4.6) with (4.8) reproduces the known formula of the currents corresponding to the Chevalley generators of $\mathcal{G}=\operatorname{sl}(n)$ in the principal case.

We wish to note that the redefinitions of the momenta that appear in $(4.9)$ - which can be absorbed into redefinitions of $\Omega^{\beta}$ in (4.6) leading to a trivial ambiguity in the solution for $\Omega^{\beta}$ - are particular quantum canonical transformations that preserve the OPEs in (4.2). Analogous canonical transformations of the momenta at fixed coordinates exist already at the classical level, and actually no ambiguity arises from the quantization.

More general canonical transformations of the quantum fields $q_{\alpha}, p^{\beta}$ can be used to relate quantizations of the classical Wakimoto realization in different systems of coordinates on $G_{-}$. Two systems of coordinates $q_{\alpha}$ and $Q_{\alpha}$ on $G_{-}$may be called 'polynomially equivalent' if the change of coordinates $q_{\alpha}=q_{\alpha}(Q)$ is given by polynomials. It is shown in [13] that with any polynomial change of coordinates on $G_{-}$one can associate a canonical transformation of the corresponding set of quantum fields, $\left(q_{\alpha}, p^{\beta}\right) \Leftrightarrow\left(Q_{\alpha}, P^{\beta}\right)$. Such a canonical transformation naturally induces a quantization of the classical Wakimoto realization in any system of coordinates which is polynomially equivalent to an upper triangular system of coordinates. Conversely, the quantizations obtained in polynomially equivalent systems of coordinates are always related by quantum canonical transformations. Finally, it is also demonstrated in [13] that the quadratic form of the affine-Sugawara stress-energy tensor given in (4.10) is maintained under the polynomial changes of coordinates.

It is clear that the polynomiality of the coordinates is necessary for the polynomiality of $I\left(q, p, j_{0}\right)$ in (4.6). We see from the above remarks that upper triangularity is a sufficient but not a necessary condition on the 'admissible' polynomial coordinates in which the quantization of the classical Wakimoto realization can be performed. We believe that all such admissible systems of coordinates are polynomially equivalent, but did not prove this yet. 


\section{Conclusion}

Wakimoto realizations play an important role in the representation theory of affine Lie algebras as the building blocks of resolutions of irreducible highest weight representations [3, 4, 5]. Furthermore, they can be used to compute correlation functions in the WZNW model [3, 4, 5, 6], and to obtain free field realizations of $\mathcal{W}$-algebras [7, 19, 20, 21]. The so-called screening charges play a crucial role in all these applications. Screening charges are operators that commute with the Wakimoto current, and can be used to build intertwiners between different highest weight representations of the affine Lie algebra. With a compact explicit expression for the Wakimoto current at our disposal, it is possible to explicitly construct the screening charges as well. Below we summarize the main ideas of the construction, referring to [13] for details and for further discussion.

In keeping with the spirit of the preceding sections, we wish to define the classical version of the screening charges first. For this we introduce a $G_{0}$-valued field $h_{0}$ which is related to the current $j_{0}$ by

$$
j_{0}=K h_{0}^{-1} h_{0}^{\prime} .
$$

One should be careful not to confuse $h_{0}$ with the coordinate $g_{0}$ on the constrained phase space that appears in Section $3 ; h_{0}$ is in general a multiple-valued function on the circle. In terms of $h_{0}$, we define the charges $S_{\xi}$ by

$$
S_{\xi} \equiv \int_{0}^{2 \pi} d \sigma \operatorname{Tr}\left(\xi h_{0} j h_{0}^{-1}\right) \quad \forall \xi \in \mathcal{G}
$$

We then find that $S_{\xi}$ commutes with the classical Wakimoto current $I$ in (3.2) if and only if

$$
\xi \in \mathcal{G}_{-} \cap\left[\mathcal{G}_{+}, \mathcal{G}_{+}\right]^{\perp}
$$

The $S_{\xi}$ with $\xi$ in (5.3) constitute the classical screening charges of the Wakimoto realization.

We wish to subsequently quantize the classical screening charges. We here restrict ourselves to the principal case, for which $\mathcal{G}_{0}$ is the Cartan subalgebra. In this case, we can bosonize $j_{0}$ simply by putting $j_{0}=-i \sqrt{y} \partial \phi$, where $\phi$ is a $\mathcal{G}_{0}$-valued scalar field with the OPE

$$
\operatorname{Tr}\left(X_{0} \phi\right)(z) \operatorname{Tr}\left(Y_{0} \phi\right)(w)=-\operatorname{Tr}\left(X_{0} Y_{0}\right) \log (z-w) \quad X_{0}, Y_{0} \in \mathcal{G}_{0}
$$

and $y=\frac{1}{2}|\psi|^{2}\left(k+h^{*}\right)$ as before. In addition, we assume that the basis $V^{\alpha}$ of $\mathcal{G}_{+}$has been chosen to coincide with root eigenspaces, in particular $V^{\alpha_{l}}$ is proportional to the step operator $E_{\alpha_{l}}$ for every simple root $\alpha_{l}$. Noticing that at the classical level $h_{0}=\exp \left(-\frac{i}{\sqrt{K}} \phi\right)$ and that the space in (5.3) is now spanned by the $E_{-\alpha_{l}}$, we obtain the following screening charges at the quantized level:

$$
S_{l} \equiv \oint \frac{d z}{2 \pi i}\left(p^{\beta}\left(\left(N_{\alpha_{l} \beta}^{-1}: \exp \left(-\frac{i}{\sqrt{y}} \alpha_{l}(\phi)\right):\right)\right) \quad l=1, \ldots, \operatorname{rank}(\mathcal{G})\right.
$$

This formula for the screening charges has been known (see e.g. eq. (3.12) in the third article in [5]), although in a less detailed form and, to our knowledge, without a complete proof 
except for $\mathcal{G}=\operatorname{sl}(n)$. With our explicit formulas the proof that the $S_{l}$ in (5.5) indeed commute with the Wakimoto current is just a direct calculation.

One can use the principal Wakimoto realization to obtain a free field realization of the $\mathcal{W}$-algebra associated with the principal embedding of $s l_{2}$ in $\mathcal{G}$, sometimes denoted as $\mathcal{W}[\mathcal{G}]$. This is done by performing a quantum Drinfeld-Sokolov reduction of $\widehat{\mathcal{G}}_{k}$ [19, 22], but now using the Wakimoto realization of $\widehat{\mathcal{G}_{k}}$ rather than $\widehat{\mathcal{G}}_{k}$ itself. The computation of the BRST cohomology is very easy, and one finds that the only object that survives is the $\mathcal{G}_{0}$-valued scalar $\phi$. In addition, the screening charges of $\widehat{\mathcal{G}}_{k}$ in (5.5) are cohomologous to

$$
\tilde{S}_{l} \equiv \oint \frac{d z}{2 \pi i}: \exp \left(-\frac{i}{\sqrt{y}} \alpha_{l}(\phi)\right):
$$

leading to a description of $\mathcal{W}[\mathcal{G}]$ as the centralizer of the screening charges of $\mathcal{W}[\mathcal{G}]$ in $(5.6)$ acting on the algebra generated by the free scalar $\phi$.

Whereas previously several of the above statements could only be analyzed by indirect methods, the explicit expressions presented in this letter allow for straightforward computations, which we expect to prove useful in future applications of the Wakimoto realizations.

Acknowledgements. JdB is supported in part by the National Science Foundation grant PHY93-09888. L.F. wishes to thank I. Tsutsui for encouragement.

\section{References}

[1] M. Wakimoto, Commun. Math. Phys. 104 (1986) 605.

[2] F.A. Bais, P. Bouwknegt, K. Schoutens and M. Surridge, Nucl. Phys. B304 (1988) 348.

[3] B.L. Feigin and E.V. Frenkel, Usp. Mat. Nauk. 43 (1988) 227 (in Russian), Russ. Math. Surv. 43 (1989) 221; Commun. Math. Phys. 128 (1990) 161; Lett. Math. Phys. 19 (1990) 307; p. 271 in: Physics and Mathematics of Strings, eds. L. Brink at al, World Scientific, 1990; E. Frenkel, Free field realizations in representation theory and conformal field theory, preprint hep-th/9408109.

[4] D. Bernard and G. Felder, Commun. Math. Phys. 127 (1990) 145.

[5] P. Bouwknegt, J. McCarthy and K. Pilch, Phys. Lett. B234 (1990) 297; Commun. Math. Phys. 131 (1990) 125; Prog. Theor. Phys. Suppl. 102 (1990) 67; p. 407 in: Strings and Symmetries 1991, eds. N. Berkovits et al., World Scientific, Singapore, 1992.

[6] H. Awata, A. Tsuchiya and Y. Yamada, Nucl. Phys. B365 (1991) 680.

[7] M. Bershadsky and H. Ooguri, Commun. Math. Phys. 126 (1989) 49.

[8] A. Gerasimov, A. Morozov, M. Olshanetsky, A. Marshakov and S. Shatashvili, Int. J. Mod. Phys. A5 (1990) 2495; A. Morozov, JETP Lett. 49 (1989) 345. 
[9] M. Kuwahara and H. Suzuki, Phys. Lett. B235 (1990) 52; M. Kuwahara, N. Ohta and H. Suzuki, Phys. Lett. B235 (1990) 57; Nucl. Phys. B340 (1990) 448; N. Ohta and H. Suzuki, Nucl. Phys. B332 (1990) 146.

[10] K. Ito and Y. Kazama, Mod. Phys. Lett. A5 (1990) 215.

[11] K. Ito and S. Komata, Mod. Phys. Lett. A6 (1991) 581; K. Ito, Phys. Lett. B252 (1990) 69.

[12] B. Kostant, Lect. Notes Math. 466 (1974) 101.

[13] J. de Boer and L. Fehér, preprint in preparation.

[14] V.V. Gorbatsevich, A.L. Onishchik and E.B. Vinberg, Structure of Lie Groups and Lie Algebras, Encyclopaedia of Mathematical Sciences, Vol. 41, eds. A.L. Onishchik and E.B. Vinberg, Springer-Verlag, 1994.

[15] R. Abraham and J.E. Marsden, Foundations of Classical Mechanics, second edition, The Benjamin/Cummings Publishing Company, 1978.

[16] E. Witten, Commun. Math. Phys. 92 (1984) 455.

[17] J. Harnad and B.A. Kupershmidt, J. Geometry and Physics 16 (1995) 168.

[18] For a review, see e.g. R.W. Gebert, Int. J. Mod. Phys. A8 (1993) 5441.

[19] B.L. Feigin and E.V. Frenkel, Phys. Lett. 246B (1990) 75; Int. J. Mod. Phys. A7 Supplement 1A (1992) 197.

[20] E. Frenkel, 'Affine Kac-Moody algebras at the critical level and quantum DrinfeldSokolov reduction', Harvard University PhD thesis, 1991; p. 433 in: New Symmetries in Quantum Field Theory, eds. J. Fröhlich et al, Plenum Press, New York, 1992.

[21] J. M. Figueroa-O'Farrill, Nucl. Phys. B343 (1990) 450.

[22] J. de Boer and T. Tjin, Commun. Math. Phys. 160 (1994) 317 